the climate-change problem for each scenario: take the anticipated carbon emissions and subtract an emissions profile designed to bring about a specific goal - say, a significant chance of keeping the increase in global average temperature at or below $2^{\circ} \mathrm{C}$. The result is the amount of carbon emissions that must be avoided to reach the goal. This figure, typically hundreds of billions of tonnes of carbon over the century, is then treated as the challenge that must be met by new energy technologies and efficiency drives.

But this approach brings with it a risk of double counting. The 'business-as-usual' scenarios actually assume that economies will somehow steadily increase their energy efficiency and decrease the amount of carbon they emit per unit of energy. The scenarios don't specify what technologies will be used to achieve these things, so unwary users could wrongly consider all reductions in either measure as progress over the status quo scenario. As a result, the amount of decarbonization required to achieve a stated goal is far larger than is often assumed.

The challenge is daunting enough without such misprision. An ever greater amount of world domestic product is coming from developing economies where the amount of energy used and carbon emitted per unit of economic activity is growing.

The history of the industrialized nations does suggest that there would, over time, be progress in energy technology and efficiency even without any threat of climate change. But global policy must now aim to increase this progress greatly, which means we have to accept that there are no meaningful 'business-as-usual' scenarios. The required progress requires new incentives, new research and, crucially, new costs imposed on the users of fossil fuels. It would also benefit from new economic models that, unlike the ones used with current scenarios, can show how the various policies aimed at replacing fossil fuels might work.

We also need to accept that the challenge of providing humanity with more energy in a minimally damaging way is even bigger than is usually thought. A simple calculation illustrates the point. If a growing population is to have access to the energy needed to drive development, then in 50 years the world's energy supply will need to at least double, even if energy use in tomorrow's great powers never rises to the per capita level seen in industrialized nations today, and if use in those nations is severely curtailed by efficiency improvements.

At the same time, if the world's climate is to be stabilized, carbon emissions from fossil fuels will have to be reduced dramatically in comparison with today's levels. To satisfy both those requirements, the amount of energy generated without the emission of fossil carbon needs to increase by something like a factor of ten.

That is the most daunting challenge humanity has ever sought to meet with a united front. The chances of success will be helped by models that allow different strategies to be compared. But no amount of scenario planning can replace the need for the will and leadership.

\section{Mismanaged measures}

\section{Surrogate end points can be helpful in clinical trials - but only if they are used with care.}

/ $/$ V hat gets measured, gets managed" is an adage that doctors know all too well. Physicians routinely monitor diseases by measuring biological factors such as cholesterol or blood pressure. And clinical trials are starting to use such indirect measures as 'surrogate markers' of a treatment's effect on the disease in clinical trials (see page 510). The practice has helped to get drugs to market quickly by allowing researchers to perform rapid and easy measurements rather than waiting for long-term clinical outcomes such as heart attacks or strokes.

But every so often, a surrogate deceives, as in the unexpected results from two recent clinical trials. One found that the drug ezetimibe, which lowers cholesterol levels, did not seem to slow the steady march of atherosclerosis in patients with high cholesterol (J. J. P. Kasterlein et al. N. Engl. J. Med. 358, 1431-1443;2008). In the other, one part of the trial was halted when the researchers found that using a combination of strict diet and insulin to lower diabetics' blood-sugar levels to those of healthy people actually increased mortality rates in some patients.

These results conjure up unpleasant memories of past surprises with surrogate markers. Several drugs that quelled irregular heartbeats in patients who had had a heart attack shocked cardiologists when they were found also to increase the risk of a subsequent heart attack. Another candidate drug for controlling cholesterol, torcetrapib, raised levels of the 'good' cholesterol - high-density lipoproteins - yet increased the number of deaths. Such events are troubling, and regulators face a high-stakes tug-of-war between the risk of relying on a surrogate marker, and the harm of delaying a potentially useful therapeutic from being used in the clinic.

Cardiologists at this week's annual meeting of the American College of Cardiology urged their colleagues to try other medications before resorting to ezetimibe. The warning should not have been necessary. Other cholesterol-lowering drugs have been on the market for 20 years, and have been vetted by large-scale clinical trials that evaluated direct outcomes such as heart attacks. Ezetimibe was approved five years ago in the United States solely on the basis of its ability to lower lipid concentrations. Large clinical trials to look at its effects on the cardiovascular system were not initiated until several years after it was approved. Some of the results aren't due until 2011. The choice between ezetimibe and the older drugs should have been clear, yet the statistics suggest that many doctors favoured ezetimibe. In 2006, ezetimibe accounted for $15 \%$ of the prescriptions for cholesterol medication in the United States. Sales of the drug climbed above \$5 billion in 2007.

Why did the medical community embrace ezetimibe with such enthusiasm? There are two likely explanations. The first is an aggressive and effective marketing campaign. The second is a firm belief in the predictive power of a trusted surrogate. Previous cholesterol-lowering drugs had proven successful, and in heart disease as in diabetes, the belief came to be: 'the lower, the better'.

We won't know for some time whether ezetimibe works for the end points that matter: heart attack and death. But it is clearly time to check our assumptions. Surrogate end points are valuable, but are not always accurate indicators of the important outcomes. Drugs approved solely on the basis of surrogate end points come with a risk that should not be ignored, no matter how trusted the surrogate - or how persuasive the marketing campaign. 